\title{
EMPIRICISM IN ECOLOGICAL ECONOMICS: A PERSPECTIVE FROM COMPLEX SYSTEMS THEORY
}

\author{
Jesus Ramos-Martin* \\ Departament d'Economia i d'Història Econòmica, and \\ Institut de Ciència i Tecnologia Ambientals \\ Universitat Autònoma de Barcelona \\ 08193, Bellaterra, Spain \\ Tel. Number: +34 935812504 \\ Fax number: +34935812012 \\ Email: jesusramosmartin@yahoo.es
}

\begin{abstract}
Economies are open complex adaptive systems far from thermodynamic equilibrium, and neo-classical environmental economics seems not to be the best way to describe the behaviour of such systems. Standard econometric analysis (i.e. time series) takes a deterministic and predictive approach, which encourages the search for predictive policy to 'correct' environmental problems. Rather, it seems that, because of the characteristics of economic systems, an ex-post analysis is more appropriate, which describes the emergence of such systems' properties, and which sees policy as a social steering mechanism. With this background, some of the recent empirical work published in the field of ecological economics that follows the approach defended here is presented. Finally, the conclusion is reached that a predictive use of econometrics (i.e. time series analysis) in ecological economics should be limited to cases in which uncertainty decreases, which is not the normal situation when analysing the evolution of economic systems. However, that does not mean we should not use empirical analysis. On the contrary, this is to be encouraged, but from a structural and ex-post point of view.
\end{abstract}

Keywords: Ecological economics, neo-classical environmental economics, empiricism, predictive analysis, complexity, post-normal science, policy.

\section{Introduction}

Ecological economics deals with, and is related to, policy generation and, in order to do this needs numerical data about both human and natural systems. It is the goal of this paper to analyse the role of empiricism in the framework of neo-classical environmental economics and ecological economics. After doing that, the paper defends a phenomenological and ex-post analysis to deal with the complexity of modern economies, by giving some examples of empirical work already done under this view.

The concepts underlying ecological economics and neo-classical environmental economics will be outlined, to emphasise that the latter makes some strong implicit assumptions about the working of systems under its analysis (i.e. economic systems). These assumptions are compatible neither with the main characteristics of present

\footnotetext{
* Paper accepted in Ecological Economics
} 
complex environmental systems nor with the nature of economies. This is why ecological economics deals with both the problems and the systems in an alternative way.

The structure of the rest of the paper is as follows: Section 2 focuses on the conceptual structures in ecological economics and in neo-classical environmental economics from an evolutionary perspective based on the concept of time. Section 3 presents the debate about the role of policy for sustainability purposes. Section 4 presents the position of these two schools of economic thought on empirical analysis, focusing on time and evolution. With this background, Section 5 mentions some of the latest developments in empirical analysis that have been published in the field of ecological economics, and that are an example of what could be empirical analysis when dealing with complexity in ecological economics. Finally, Section 6 reaches the conclusion that a predictive use of econometrics in ecological economics should be limited to cases in which uncertainty decreases. This leads to presenting the way ahead regarding empirical analysis in ecological economics, and its relationship to policy formulation.

\section{Conceptual structures in ecological economics and in neo-classical environmental economics}

\subsection{Neo-classical economics}

Neo-classical economics focuses on the exchange of goods and services among the economic agents, such as consumers and producers, emphasising the role of consumer preferences and resources endowments, to guarantee the economy's equilibrium. As pointed out by Ruth (1993) the main characteristics of this approach are a concentration on market mechanisms, a focus on microeconomics instead of macroeconomics, static analysis (neglecting then the history of processes), linearity, and a consideration of the environment only as a given boundary. This means that the methodology developed by neo-classical economics, general equilibrium theory, guarantees the achievement of a solution in the allocation of scarce resources (Faber et al., 1996).

To understand better neo-classical economics we might think that it follows classical mechanics in its description of the economic process. That is, production, consumption, or distribution are seen as single processes that can be analysed separately to achieve not only understanding of them, but also to make possible forecasting. In the words of Georgescu-Roegen (1971, p.319), it "is a mechanical analogue". As in mechanics, economists are seeking "universal laws" that can be applied everywhere and regardless time. Once laws are defined and basic principles or axioms are accepted, then this economics must be a theoretical science, deductive, and deterministic, capable of finding unique optimal solutions.

Since neo-classical economics follows mechanics, where all processes are reversible, its equations and models are also 'time symmetric', where time is just a cardinal magnitude, which can, therefore, be added or subtracted (Beard and Lozada, 1999). At this point it is worth mentioning Georgescu-Roegen's distinction between 'time' and 'Time'. Using his own words (1971, p.135), "T represents Time, conceived as the stream of consciousness or, if you wish, as a continuous succession of "moments", but $\underline{t}$ represents the measure of an interval $\left(T^{\prime}, T\right.$ ') ) by a mechanical clock" (emphasis in the original). Neo-classical economics claims the theory to be valid in all societies, 
that is, to be a-historic, because they are considering mechanical time, instead of historical Time. This distinction is relevant since is related to Prigogine's (Nicolis and Prigogine, 1977; and Prigogine and Stengers, 1984) Second Arrow of Time, which in words of Proops (1983: 357), is "the tendency of certain systems to become more complex and more structured".

Neo-classical natural resource and environmental economics, deals with the environment by analysing the threats of scarcity and pollution using the ideas described above. The methods developed have been: (i) optimisation in the case of managing natural resources (either renewable or exhaustible), and (ii) assigning property rights on pollution (or more generally externalities) in order to incorporate them into the price system, and thus, in the decision process under the market mechanism. This is why supporters of this approach are usually optimistic when dealing with environmental problems. For example, in the case of exhaustible resources they propose substitution between production factors, neglecting two basic issues. On the one hand, there are services provided by nature that are not substitutable at all (like the water or the carbon cycles). On the other hand, "from a physical perspective substitution cannot replace energy completely (including the energy of labour) because each factor of production depends ultimately on an input of net energy for its own production and maintenance" (Hall et al. 1986, p.46). It could be added that we can interpret the relationship between energy and matter, or any kind of production factor, as largely that of complementarity rather than substitutability.

All of these characteristics of neo-classical economics, and its environmental branch, led to it being viewed as having difficulties dealing with new and complex problems, such as environmental problems. As Clark et al. (1995) pointed out, the mechanical character of economic models does not allow them to treat evolution or structural changes in the system. This fact led to new approaches as those proposed by ecological economics.

\subsection{Economies as complex adaptive systems}

Economies are complex adaptive systems, that is, composed of large and increasing number of both components and of the relationships between them. Economies are also teleological systems (they have an aim, or end, the telos), but in a different way than non-human systems, which have only that of self-maintenance and development of the systems; economies incorporate new tele, those of the human beings belonging to the system, and they are capable of incorporating the guessed consequences of their fulfilment into the present decisions and definitions of new tele; they are thus anticipatory. They also learn from mistakes and from present developments, and they react, by changing both the actions undertaken and the tele defined; they are thus selfreflexive. They also have the ability to adapt to new changing boundary conditions (a property also shown by non-human systems), but they may consciously alter the boundary conditions. This is why the economy, as a human system, can be understood as a complex, adaptive, self-reflexive, and self-aware system (see Kay and Regier, 2000, for more details).

When analysing their structure, economic systems are nested hierarchical systems. In the case of economic systems, we can distinguish several subsystems within them, and every sector may be split into different industrial 'types' (sharing common features) and so on. The various levels of an economy exchange human activity and 
energy between them, reflecting the interconnected nature of those systems (the output of one sector enters other sector as input, and vice versa). That is, "downward and upward causation imply feedback between different levels of description in the hierarchy $(. .$.$) [then], in mathematical terms it implies additional complexity and non-$ linearity such that an economic equilibrium is no longer evident and certainly cannot be easily calculated" (van den Bergh and Gowdy, 2003).

Thus, the increased complexity of economies, their nested hierarchical nature, and the fact that they show adaptive and evolutionary behaviour, gives rise to two parallel outcomes. One is the non-linear behaviour, even chaotic behaviour that these systems show. This is a short run process that involves a given structure and the difficulty in comprehending it by using the traditional methods of analysis based on hard (quantitative) modelling. The other is the emergence of novelty, which is long run, and involves changes in the structures. An alternative way of presenting this is by using the concepts of phenotypic evolution (different realisations of potentialities of the systems, which are susceptible of prediction) and genotypic evolution (emergence of new institutions or techniques, which by definition are unpredictable; that is, new potentialities) (Faber and Proops, 1998). Therefore, they are contextual by nature, depending on one particular space and time scale, and so they have to be analysed, taking one specific time and space scale into account. Since the parameters do change, this fact brings uncertainty up to the scene in the form of the selection of the set of parameters to be used to characterise the structure of the system. That is, the question of who decides the set of parameters is relevant here. Moreover, because of the same characteristics, and because being anticipatory systems means that they incorporate possible future states into the present, there may be multiple equilibria.

If this is the case and we cannot find regularities or periodicity in the evolution of the systems, they would be largely unpredictable. This is so because when the system suffers a sudden change that makes it move away from one attractor point to other thanks to a random fluctuation, a small change in the parameters may have large consequences (Gleick, 1987). This is the so-called Butterfly Effect, later called 'sensitive dependence on initial conditions'. If this is happening, then very small influences can no longer be neglected; this makes modelling and prediction hard. In this sense we must consider the future to be open and uncertain under a non-linear world (Haken and Knyazeva, 2000).

However, this chaotic behaviour gives rise to new ordered structures within systems that can be approached from complex systems theory. In the words of Haken and Knyazeva (2000, p.59), "although the future is open, and there are a number of possible evolutionary ways for a complex system, not any arbitrary (either conceivable or desirable) evolutionary way is feasible in a given system". This is so because history counts and once a path is taken, some others are closed forever (i.e. path dependency). This reduces the number of possible attractors, and it induces, again, non-linear behaviour in the development of the system. It also reflects irreversibility. One of the ways of improving the knowledge in that context is by finding historical regularities that may reflect the emergent properties of the systems. However we have to bear in mind that even if some regularities are found, there might not be any causal relationship between the processes or variables. Even, "it is also possible that no regularities will appear despite the existence of causal relations between the factors being studied" (Ramsay, 1998, p.168, emphasis in the original). 
Thus, even though the future is open, only some of the evolutionary paths are feasible and can develop, giving rise to different attractors. Haken and Knyazeva (2000) put it in these terms, "only the structures being in accordance with the inner evolutionary trends of the systems can arise. And nothing else but select meta-stable structures can be constructed in the system. These are a kind of evolutionary laws of prohibition" (emphasis in the original). These laws tell us that we cannot have equilibrium, and that the meta-stable structure has to be compatible with the internal constraints of the system. However, contrary to neo-classical economics, these do not tell us anything about what kind of systems or solutions we are going to have.

\subsection{Ecological economics}

Ecological economics takes production, or the transformation of energy and materials, as one of its focal points, as it was done by classical economic thought, but it uses in its analysis the insights derived from thermodynamics, i.e. the second law of thermodynamics that introduced the issue of irreversibility. It is, then, an evolutionary science. An evolutionary science deals with historical events, and the processes between the events; that is, it deals with the issue of time. Using Georgescu-Roegen's distinction about time, it can be said that an evolutionary science deals with historical 'Time', whereas neo-classical economics deals with mechanical 'time', so neoclassical economics cannot be considered as an evolutionary science.

Ecological economics also deals with new complex adaptive systems, as presented above. Ecological economics, thus, unlike neo-classical environmental economics, focuses, among other things, on evolution of economies, on the process of becoming, on structural change, and the emergence of novelty (in the form of technological change, for example), all features shown by complex adaptive systems. The presence of novelty, the feedback mechanisms between the different levels of the hierarchy, and their anticipation, ensure that uncertainty is always present when dealing with these systems. This is one reason to ask for a new epistemology, as it is done in the next section. In fact, the more research we apply, the more uncertainty is generated, new questions arise, and new relationships between variables are found. In words of Faber and Proops (1998, p.110) when talking of environmental problems, "very often they involve the emergence of unpredictable events (novelty) (...) this implies that the simple sequence of problem $\rightarrow$ science $\rightarrow$ technique $\rightarrow$ solution is not necessary valid. On the contrary, we experience that our increasing knowledge may even impede the investigation for solutions". This fact causes the issue of unpredictability, very often present when analysing environmental problems, and therefore relevant for ecological economics, and especially for policy generation.

\section{The role of policy}

In economics, the role of policy is viewed differently depending on the school of thought taken. Neo-classical environmental economics conceives of the existence of policy based in economic analysis. It analyses market failures that induce environmental externalities, and tries to design policy to 'correct' these failures, and eventually give optimality. To do that, it uses the tools explained before in Section 2.1 .

However, the new environmental problems are characterised by the following traits: facts are uncertain, there are values in dispute, the stakes are high and decisions are 
urgently needed (Funtowicz and Ravetz, 1991), as we saw in Section 2.2 when dealing with complex economies. In this context, ecological economics defends a new epistemology to deal with complexity. So, in this context dominated by uncertainty and ignorance (we do not know what we do not know), a new approach to tackle these problems is needed. This approach has been called "poststructural" or "post-modern" (Denzin, 1994), "civic science" O’Riordan (1996), or "post-normal science" (Funtowicz and Ravetz, 1991). Ecological economics is said to be an example of postnormal science (Funtowicz and Ravetz, 1994).

In this approach it is not said that present scientific knowledge is no longer valid or applicable, but rather, that there exist some emergent problems characterised by complexity and uncertainty in which the "normal" science can not be used with the traditional methods alone (i.e. the sequence of problem $\rightarrow$ science $\rightarrow$ technique $\rightarrow$ solution mentioned above).

In post-normal science it is admitted that objective reality can never be captured because of the inherent changing characteristics of the systems analysed, and that research is influenced by values of the researcher and, therefore, there is no value-free science (Denzin and Lincoln, 1994; Prigogine and Stengers, 1984). With this background, policy-making becomes a multidimensional and multifaceted process (Rist 1994) in which research is only one source of knowledge among others (such as common sense, beliefs, etc.) that seek to influence the final result.

In post-normal science, research and knowledge have not the intention of providing policy-makers with a solution to the problem avoiding the need for them to take the political decision, and legitimating all of their acts. Rather, the idea is to create a shared contextual understanding about the issue (Rist 1994) in such a way that we keep informed all of the actors involved in the process of decision-making, but letting them reach a satisfactory compromise solution. This compromise solution will not have the aim of being a reflection of 'truth', but rather it will be a socially constructed view of reality (Clark et al. 1995), an agreed understanding of both the problem and the ways of tackling it.

As Kay et al. (1999, p.737) said, "The program of post-normal science is to provide a basis for the understanding necessary to unravel complexity (emergence, irreducible uncertainty, internal causality), so that we may successfully anticipate, when possible, and adapt, when appropriate or necessary, to changes in the self-organizing systems of which we are an integrated and dependent part".

Post-normal science is, thus, about assuming that in both science and the process of decision-making there exist value judgements, and it is proposed, therefore, that we have to guarantee the quality of the process of decision-making rather than the final result, because there is no objective truth (Funtowicz and Ravetz, 1994). To do that, we should shift from a substantive or result-oriented rationality to a new procedural rationality (Simon, 1983), in which the process of knowledge generation is the relevant issue instead of the focus being on the final outcome. Such procedural rationality would imply an extension of the peer review community to people from other disciplines and to people affected by the issue. The work would be to manage the uncertainty that characterises every field to get the highest quality information we can achieve (Funtowicz and Ravetz, 1994). "This extension of the peer community is 
essential for maintaining the quality of the process of resolution of problems of reflexive complex systems" (Martinez-Alier et al., 1998, p.282).

In sum, in ecological economics, as a post-normal science, policy is thus seen as a social steering mechanism implying the need to engage all stakeholders in reaching consensus.

\section{Empirical analysis under complexity}

As noted by Ramsay (1998), empiricism is based on the idea that knowledge of the world is generated by experience rather than by reason. However, inside empirical analysis we shall focus here only in two main branches, the positivist approach, and the phenomenological (or interpretivist) approach.

The positivist approach tries to use the "scientific method" by deducing theories as a result of formulating and testing hypotheses based on statistical data analysis. It formulates hypotheses on cause-effect relationships and tests them. If they pass the tests, this is the basis for a future generally applicable law generated by induction. This approach assumes that the subject of the study, i.e. the functional relations that define the relationships between the variables describing the system, are uniform and unchanging. For example, Heckman (2001, p.3), notes, "empirical research is intrinsically an inductive activity, building up generalizations from data, and using data to test competing models, to evaluate policies and to forecast the effects of new policies or modifications of existing policies". Under this partial view on empiricism it is very difficult to account for change and evolution.

The phenomenological approach, on the other hand, takes a different view of the subject under analysis than the positivist one. It acknowledges that when dealing with human systems, these have the intrinsic characteristics of changing and evolving in time; that is, the characteristic of becoming, due to external factors (i.e. shocks) or to internal causes, such as changes in preferences, technologies, or institutions (i.e. genotypical evolution as explained in Section 2.2). This fact makes it impossible to consider them as uniform and unchanging, so, in order to explain them, we have first to understand them.

Neo-classical environmental economics defends a position favourable to the use of predictive analysis and thus to the positivist approach. It defends the notion that expost analysis can give insights about the structures of the systems, and by extrapolating them into the future, can generate an ex-ante prediction of the development of variables, which can then be used for policy. In particular, neoclassical environmental economics supports an ex-post analysis for ex-ante predictions because is implicitly based in classical mechanics where that is possible. This is because the basic characteristics of physical systems are described by universal laws; that is, they are not subject to structural change (i.e. gravity is stable, and so on). But this is not the case with biological systems and, in particular, human systems, where the underlying characteristics of systems, and therefore the parameters that we use to describe those characteristics, are constantly evolving, making prediction much more problematic (Faber et al., 1996). So, neo-classical environmental economics would be extrapolating past results into the future by assuming two things; one, that the parameters defining both the system and the relationships between the different variables do not change in time; and two, that the 
functional relationship between the variables also remains stable for the period of time being predicted. For modern economic systems, these assumptions seem not to apply, since systems are constantly evolving and in the process of becoming, and therefore, if we want our representation of them to be updated, both the parameters and the functional relationships between them should evolve as well.

Ecological economics, therefore, can be considered as representative of the phenomenological approach. Since it deals with complexity, and complexity is characterised by irreversibility and stochasticity (Prigogine, 1987), it concludes that linear deterministic models are ineffective. And since it is an evolutionary science, it defends that all understanding regarding a system or a process is context dependent.

\section{Recent empirical work in the field of ecological economics}

With this background on how the conceptual structures of both neo-classical environmental economics and ecological economics can be defined, and with the different roles of policy and empirical analysis that each discipline defends, the next step is to proceed with an exemplification of the kind of empirical work that is suggested to be carried out in ecological economics when dealing with complex systems.

Some of the work published in the field of ecological economics deals with complex systems in a simple way, for example by assuming constancy of the structure of agents' preferences (neglecting irreversibility or the history of processes). Some assume linearity and constancy in both the parameters and the relationships between the variables defining the systems; that is, stability in the genotypes. With this analysis, they can recommend policies based on the results of their projections, that is, based in the extrapolation of past results. Then we can say that 'science' seeks to 'model' the genotype so it can predict the phenotype. But, scientific data is only on the phenotype (the realisation or representation of the potentiality of that system). So, if the phenotype changes, observations on phenotypes are a poor basis for modelling and prediction. This is what is happening with an important portion of empirical work in ecological economics, that they are not matching the technique to the problem analysed. They are not keeping updated the set of parameters and functional relationships to the changes in the genotype or the basic characteristics of the systems; that is, to their evolution or process of becoming. One could think, therefore, there is not much difference with the methods applied by neo-classical environmental economics.

There is, however, at least another way of understanding empirical analysis in ecological economics. Perrings and Walker (1997) use a model of resilience and empirical analysis to explain the importance of fire in the self-organisation of semiarid rangelands, being a vehicle of a destructive creation phase. That is, they explain the role of fire as a trigger of the shifting of the system from one meta-equilibrium to another. Another example is that of Jackson and Marks (1999), where the authors analyse the past distribution of consumer expenditure in the UK for a period of time, identifying some patterns of behaviour with different consequences upon the environment that can be accounted for when deriving policy. However, one of the topics in which this kind of analysis has been more successful is that of the environmental Kuznets curve, because it relates the evolution of income (and therefore of the economy) with some physical variables such as energy consumption 
or use of materials. The environmental Kuznets curve looks at first sight as a hypothesis which can be retained or discarded according to relatively simple econometric work. Some authors find an inverted U pattern, i.e. an improvement in (some) environmental indicators at sufficiently high levels of income, and then conclude with an ex-ante prediction that growth is good for (at least part of) the environment. Some authors find not an inverted U curve but rather an N, implying deterioration at still higher levels of income. Other authors find more complicated patterns (with "attractor" points) (see Rothman (1998), Suri and Chapman (1998), Unruh and Moomaw (1998) or De Bruyn and Opschoor (1997)). This looks as a debate among equation-fitting empiricists. My point is that it is not, in the following sense. The authors I praise here, include beyond the equations, a narrative in which punctual technological changes (say, from coal to gas), or environmental policy (onetime price increases like for oil in 1973, or the introduction of an eco-tax), or a cultural change in consumer or public awareness of pollution, may play roles in the shape of the EKC. Then, their explanations resemble more those of socio-economicenvironmental historians; they become complex and contextual, with little predictive contents.

Recently, another group of papers dealing with societal metabolism have tackled the issue of complexity in economic systems with an alternative methodology. In particular, the papers use a new approach, called Multiple-Scale Integrated Assessment of Societal Metabolism (MSIASM), in relation to sustainability of human society. A detailed presentation of theoretical aspects, a numerical validation, and applications in the form of case studies have been presented elsewhere (Giampietro and Mayumi, 2000a,b; Pastore et al., 2000; Ramos-Martin, 2001a; Falconi-Benitez, 2001; Gomiero and Giampietro, 2001). In particular, Ramos-Martin (2001a), extending some research initiated before (Ramos-Martin, 1999, 2001b), dealt with the historical evolution of energy intensity in Spain to respond to the debate on the environmental Kuznets curve with a counterexample. It presented first the conventional view of energy intensity in energy economics, and later an evolutionary explanation of that evolution that stressed the non-linear behaviour of the variable as a contrast with other economies. Contrary to those economies, when representing a phase diagram for Spain, it showed that the energy intensity flipped between different 'attractor' points, that is, it went through phases of stability (when moving around the two attractor points) and a transitional phase (when moving from one attractor to the other), showing that the hypothetical environmental Kuznets curve does not apply to the trajectory of energy intensity in Spain. Finally, a historical and biophysical analysis mixing different kinds of variables (GDP, working time, and energy consumption) gave interesting explanations to the evolution of energy intensity (such as the increasing responsibility of the household sector in its increase), exemplifying the necessity for using parallel non-equivalent descriptions of the same facts when dealing with complex systems because the generation of redundancy, or 'mosaic effects', among the various pieces of information improves the robustness of the analysis and gives the possibility of getting new insights not shown by the conventional economic representation alone.

The relevant here is that all of these papers took the phenomenological approach and dealt with an ex-post understanding on how systems work, by trying to find statistical regularities that reflect the underlying characteristics of economic systems, but without any aim of predicting the future using past parameters. On the contrary, the 
aim of these papers was to explain how the system 'got there', what were the mechanisms underlying the behaviour of some key variables, such as energy consumption. This is why I think they are an example of the kind of empiricism I understand should be applied when dealing with environmental problems that belong to the realm of post-normal science in open complex economic systems. This particular type of methodology fits better with the characteristics of complex economic systems as presented above in Section 2.2.

\section{The way ahead}

The criticism presented here on the use of the positivist version of empirical analysis does not mean that we cannot conduct some forecasts about the future behaviour of the variables. We can do it, provided that we are analysing the variable or the system when they are near or at, one attractor point (i.e. they are meta-stable) or when they are following a well-established trend identified historically. In these cases, when the level of uncertainty decreases, prediction is possible, under certain limitations (a sudden change is always possible). However, when the system is at a bifurcation point (i.e. in the very moment of shifting from one attractor point), prediction is not possible because we might have novelty expressed either by an external shock or by internal causality, which will drive the system towards one attractor or other. For example, internal causality may be caused by feedback loops between the different hierarchical levels of the system. We should bear in mind that when the differences in scale are too large, it is almost impossible to relate the non-equivalent information obtained from the different levels, making prediction almost impossible. This is a reflection of the unavoidable indeterminacy of the representation of these systems across scales (Mandelbrot, 1967).

So, if a basic characteristic of complex systems is that "they can only be approximated, locally and temporarily, by dynamical systems" (Rosen, 1987, p.134), but we still try to control them by using predictive dynamic models, we may face a "global failure" (Rosen, 1987, p.134, emphasis in the original) in the form of a growing discrepancy between what the system is doing and what the model predicted. This is one of the reasons why normal science is losing credibility among citizens, and why post-normal science, with its interest not in finding 'truth' but on giving good quality information for the decision-making process, is viewed as a way out of that difficulty.

When analysing data, in order to tackle complexity we can adopt the idea of triangulation (Ramsay, 1998) or parallel non-equivalent descriptions (Giampietro and Mayumi, 2000a). This idea consists of using more than one source for the data, analysing the data with different theories or models, or using different hierarchical levels at the same time, in order to gain robustness in our analysis and give more credibility to scientific analysis. This will bring redundancies, which are rather positive since they will reinforce the argument or the regularities that we may find. This is thus an argument in favour of a inter-disciplinary approach to sustainability, in which the different readings of the different disciplines are seen as compatible in generating the overall understanding of the structure of the system, and its development.

If the use of empiricism for prediction by econometrics (i.e. time series analysis) is very limited, what kind of empiricism can we use? In ecological economics we are 
interested in evolution, the process of becoming, structural change and the emergence of novelty; therefore, first we have to bear in mind that since stochastic processes are dominant in nature, scientific theories should be more down-to-earth, based in direct observations. Then, we should use empirical analysis not to give the exact values of the parameters in future, but to discriminate between those theories which are consistent with reality and those which are not. In cases of high uncertainty we should, therefore, describe and understand instead of seeking to explain and predict. Hence, we would be closer to the generation of scenarios by using narratives (i.e. soft modelling) rather than to forecasting (i.e. hard modelling). This is so because the nature of evolutionary complex adaptive systems, characterised by irreversibility and stochasticity, with their numerous possible trends, their uncertainty, the emergence of novelty, makes them largely unpredictable. That is, ex-ante modelling is often not useful for policy. We have to admit that there are no deterministic explanations (universal and a-historical). Rather we can describe and understand these systems by finding historical and spatial, regularities, and by looking at the emergence of such systems' properties. This leads us to admit that the knowledge we can obtain from complex systems is context dependent (Clark et al., 1995); it is dependent on the time window considered and also on the spatial context. This is the reason why, as pointed out by Boulding (1987), the failure in our predictions are not the responsibility of human knowledge itself. Rather, it reflects an inherent property of complex systems, that of unpredictability. Therefore, our failure might come either because we do not know the parameters of the system (ignorance) or because they change very rapidly (emergence of novelty, evolution) reflecting structural or genotypical change caused by external shocks or by internal causality within systems (e.g. chaotic behaviour).

Science applied to the decision-making process under the post-normal science framework would then be limited to assessing the consequences of the different policies, and to providing a phenomenological narrative or interpretation of how the future might unfold (Kay et al., 1999); This is part of the process of guaranteeing transparency and fairness in the process of decision-making, by promoting a continuous dialogue with stakeholders and policy makers. Thus, "these narratives focus on a qualitative/quantitative understanding which describes:

- The human context for the narrative;

- The hierarchical nature of the system;

- The attractors which may be accessible to the system;

- How the system behaves in the neighbourhood of each attractor, potentially in terms of a quantitative simulation model;

- The positive and negative feedbacks and autocatalytic loops and associated gradients which organize the system about an attractor;

- What might enable and disable these loops and hence might promote or discourage the system from being in the neighbourhood of an attractor; and

- What might be likely to precipitate flips between attractors" (Kay et al., 1999, p.728).

The implication of the argumentation presented before is that the evolution of complex systems is not fully predictable. This fact leads us, when dealing with sustainability, to the issue of incommensurability of values as a key characteristic that should distinguish ecological economics from environmental economics (MartínezAlier et al., 1998). 
Thus, the fact that the future is open has some repercussions from a policy perspective. This openness asks for what has been called 'soft management' by Haken and Knyazeva (2000). This has to be understood as encouraging flexibility in response to changing boundary conditions. This flexibility can be achieved by enhancing the diversity in the system. The more diversity, the more responses we will have to changing conditions, with more chances that one, or some of these responses, will be successful and will bring the system ahead in its development. That is, diversity increases the adaptive capacity of the system. This is something that Holling (1996), following Walker et al. (1969), called 'ecological resilience', in contrast to 'technical resilience' which would be closer to finding and returning to the initial equilibrium.

In conclusion, in complex systems prediction is very often not possible not only because the parameters defining the relationships between variables may change (phenotypic evolution), but also because the functional relation itself may also change (genotypic evolution) since they are involved in the process of becoming of the system, generating therefore more novelty. Consequently, a predictive use of econometrics (i.e. time series analysis) in ecological economics when dealing with complex systems should be limited to those non common situations in which uncertainty decreases. Rather, the phenomenological approach presented here, and exemplified by the papers mentioned in Section 5 dealing with an ex-post analysis, seems more suitable for ecological economics to deal with the issue of evolution of complex systems such as economies, involving novelty in the form of structural change. This may also include, as stated above, the use of econometric analysis to account for past developments and trends. At the end, history does count.

\section{Acknowledgements}

I would like to thank John Proops, Mario Giampietro, Jeroen van den Bergh, Roger Strand, Joan Martínez-Alier, Giuseppe Munda, and some anonymous referees for useful comments and fruitful disagreements. I would also thank the EU-funded project MCDA-RES, contract NNE5-1999-NNE5/273/2001 for funding. Naturally, none of the above is responsible for the views expressed or for any remaining errors.

\section{References}

Beard, T.R. and Lozada, G.A., 1999. Economics, Entropy and the Environment. The Extraordinary Economics of Nicholas Georgescu-Roegen. Edward Elgar. Cheltenham, UK.

Boulding, K.E., 1987. The epistemology of complex systems, European Journal of Operational Research, 30: 110-116.

Clark, N., Pérez-Trejo, F. and Allen, P., 1995. Evolutionary Dynamics and Sustainable Development: A Systems Approach. Edward Elgar. Aldershot, UK.

De Bruyn, S.M. and Opschoor, J.B., 1997. Developments in the throughput-income relationship: theoretical and empirical observations, Ecological Economics, 20: 255268. 
Faber, M. and Proops, J.L.R., 1998. Evolution, Time, Production and the Environment. Springer, Berlin.

Faber, M., Manstetten, R. and Proops, J.L.R., 1996. Ecological Economics: Concepts and Methods. Edward Elgar. Cheltenham.

Falconi-Benitez, F., 2001. Integrated assessment of the recent economic history of Ecuador, Population and Environment, 22 (3): 257-280.

Funtowicz, S.O. and Ravetz, J.R., 1991. A new scientific methodology for global environmental issues, in R. Costanza (Editor): Ecological Economics: The Science and Management of Sustainability. Columbia University Press, New York.

Funtowicz, S.O. and Ravetz, J.R., 1994. The worth of a songbird: Ecological economics as a post-normal science, Ecological Economics, 10: 197-207.

Georgescu-Roegen, N., 1971. The Entropy Law and the Economic Process. Harvard University Press, Cambridge, Massachusetts.

Giampietro, M. and Mayumi, K., 2000a. Multiple-scale integrated assessment of societal metabolism: Introducing the approach, Population and Environment, 22 (2): 109-153.

Giampietro, M. and Mayumi, K., 2000b. Multiple-scale integrated assessment of societal metabolism: Integrating biophysical and economic representations across scales, Population and Environment, 22 (2): 155-210.

Gleick, J., 1987. Chaos: Making a New Science. Penguin, Harmondsworth.

Gomiero, T. and Giampietro, M., 2001. Multiple-scale integrated analysis of farming systems: The Thuong Lo Commune (Vietnamese Uplands) case study, Population and Environment, 22 (3): 315-352.

Haken, H. and Knyazeva, H., 2000. Arbitrariness in nature: synergetics and evolutionary laws of prohibition, Journal for General Philosophy of Science, 31: 5773.

Hall, C.A.S., Cleveland, C.J. and Kaufmann, R.K., 1986. The Ecology of the Economic Process: Energy and Resource Quality. John Wiley \& Sons, New York.

Heckman, J.J., 2001. Econometrics and empirical economics, Journal of Econometrics, 100: 3-5.

Holling, C.S., 1996. Engineering resilience versus ecological resilience, in Schulze, P. (ed.): Engineering Within Ecological Constraints. National Academy of Engineering. National Academy Press, Washington DC.

Jackson, T. and Marks, N., 1999. Consumption, sustainable welfare and human needs - with reference to UK expenditure patterns between 1954 and 1994, Ecological Economics, 28(3): 421-441. 
Kay, J.J., Regier, A.H., Boyle, M. and Francis, G., 1999. An ecosystem approach for sustainability: addressing the challenge for complexity, Futures, 31: 721-742.

Kay, J.J., and Regier, H., 2000. Uncertainty, complexity, and ecological integrity: insights from an ecosystem approach, in P. Crabbé, A. Holland, L. Ryszkowski and L. Westra (Editors): Implementing Ecological Integrity: Restoring Regional and Global Environmental and Human Health, Kluwer, NATO Science Series, Environmental Security. Dordrecht.

Mandelbrot, B.B., 1967. How long is the coast of Britain? Statistical self-similarity and fractal dimensions, Science, 155: 636-638.

Martínez Alier, J., Munda, G. and O’Neill, J., 1998. Weak comparability of values as a foundation for ecological economics. Ecological Economics, 26: 277-286.

Nicolis, G., and Prigogine, I., 1977. Self-Organization in Nonequilibrium Systems. John Wiley \& Sons, New York.

O'Riordan, T., 1996. Democracy and the sustainable transition, in W.M. Lafferty and J. Meadowcroft (Editors): Democracy and the Environment. Problems and Prospects. Edward Elgar: Cheltenham.

Pastore, G., Giampietro, M. and Mayumi, K., 2000. Societal metabolism and multiple-scale integrated assessment: Empirical validation and examples of application, Population and Environment, 22 (2), 211-254.

Prigogine, I., 1987. Exploring complexity, European Journal of Operational Research, 30: 97-103.

Prigogine, I. and Stengers, I., 1984. Order Out of Chaos. Heinemann: London.

Proops, J.L.R., 1983. Organisation and dissipation in economic systems, Journal of Social Biological Structures, 6: 353-366.

Ramos-Martin, J., 1999. Breve comentario sobre la desmaterialización en el estado español, Ecología Política, 18: 61-64.

Ramos-Martin, J., 2001a. Historical analysis of energy intensity of Spain: From a "conventional view" to an "integrated assessment", Population and Environment, 22 (3): 281-313.

Ramos-Martin, J., 2001b. Non-linearity in energy metabolism of Spain: "Attractor Points" for the Development of Energy Intensity, in S. Ulgiati, M. Brown, M. Giampietro and K. Mayumi (Editors), Advances in Energy Studies. Exploring Supplies, Constraints, and Strategies, Modesti Publisher, Padova.

Ramsay, J., 1998. Problems with empiricism and the philosophy of science: implications for purchasing research, European Journal of Purchasing \& Supply Management, 4: 163-173. 
Rist, R.C., 1994. Influencing the policy process with qualitative research, in N. Denzen and Y. Lincoln (Editors.): Handbook of Qualitative Research. SAGE Publications: London.

Rosen, R., 1987. On complex systems, European Journal of Operational Research, 30: 129-134.

Rothman, D.S., 1998. Environmental Kuznets curve - real progress or passing the buck? A case for consumption-based approaches, Ecological Economics, 25: 177-194.

Ruth, M., 1993. Integrating Economics, Ecology and Thermodynamics. Kluwer, Dordrecht.

Simon, H.A., 1983. Reason in Human Affairs. Stanford University Press, Stanford.

Suri, V. and Chapman, D., 1998. Economic growth, trade and energy: implications for the environmental Kuznets curve, Ecological Economics, 25: 195-208.

Unruh, G.C. and Moomaw, W.R., 1998. An alternative analysis of apparent EKC-type transitions, Ecological Economics, 25: 221-229.

Van den Bergh, J. and Gowdy, J.M., 2003. The microfoundations of macroeconomics: An evolutionary perspective, Cambridge Journal of Economics, 27(1): 65-84.

Walker, B.H., Ludwig, D., Holling, C.S., and Peterman, R.M., 1969. Stability of semi-arid savanna grazing systems, Ecology 69: 473-498. 